\title{
The geometry-free GPS ambiguity search space with a weighted ionosphere
}

\author{
P. J. G. Teunissen \\ Delft Geodetic Computing Centre (LGR), Faculty of Geodesy, Delft University of Technology, Thijsseweg 11, 2629 JA Delft, \\ The Netherlands
}

Received: 16 July 1996; Accepted: 24 January 1997

\begin{abstract}
In this contribution the impact of a presumed presence or absence of the ionospheric delays on the quality of the least-squares ambiguities is analysed. The spatial correlation and the time correlation of the ionospheric delays are captured in an a priori ionospheric variance matrix. It is shown how the size and shape of the ambiguity search space is affected by the use of these a priori ionospheric weights. As a result an exact description can be given of the interpolatory character of the ambiguity variance matrix as function of the ionospheric weights. In order to give a qualitative analysis of the various effects, analytical rather than numerical results are emphasized.
\end{abstract}

Key words. GPS, ambiguity, ionosphere

\section{Introduction}

Estimation of the integer carrier-phase ambiguities is usually based on one of the two following single-baseline models. Either one parametrizes the double-differenced (DD) observation equations of the code and carrierphase observables in terms of the baseline components, or one parametrizes them in terms of the DD receiversatellite ranges. For most surveying applications, the first approach is the most common mode of operation; it has three advantages over the second. First, the model is directly parametrized in the baseline, which after all, is usually the parameter that one ultimately likes to solve for. Secondly, since the model is parametrized in the baseline, its design matrix captures the relative receiversatellite geometry. As a result extra strength is given to the model, in particular in the case of satellite redundancy (i.e. the tracking of more than four satellites). Thirdly, when more than one epoch of data is used, code data are not strictly needed. The change in the relative receiver-satellite geometry replaces, in a sense, the information which otherwise would have been needed from the code data.

Despite these advantages, the second approach still has its appeal. This stems mainly from its simplicity. The observation equations, for instance, are linear from the outset. Also, no particular precautions have to be taken for the tropospheric delays, since they get automatically lumped with the unknown DD receiver-satellite ranges. Thus the ambiguity estimates are known to be free from biases due to residual tropospheric delays. And as long as one is able to formulate the double differences, no special requirements are needed as to the number of satellites tracked. Thus a minimum of two satellites suffices.

In this contribution we will consider the second approach. The objective of the present study is to analyse the impact of the presumed presence or absence of the ionospheric delays on the quality of the least-squares estimators of the ambiguities. It is well known that the ionosphere decorrelates as a function of the baseline length. For sufficiently short baselines the ionosphere becomes fully correlated. As a result one may assume the DD ionospheric delays to be known or to be absent from the model. However, this assumption is not valid anymore when the baseline length increases. For sufficiently long baselines, the ionospheric decorrelation reaches its maximum and one is forced to include the ionospheric delays into the model without any informative bounds on its level of uncertainty. For the intermediate cases of baseline length though, one may use a priori weights for the ionospheric delays. The ionospheric variance matrix should then reflect the presumed uncertainty about these delays. In this contribution we will assume that this ionospheric variance matrix consists of two components. One component that reflects the spatial correlation and another that reflects the time correlation of the ionosphere. This second component is therefore directly related to the sampling rate.

In Sect. 2 we start off by defining the model of observation equations on which our analysis is based. This is followed in Sect. 3 with a brief review of the steps for computing the integer least-squares ambiguities. In 
particular the relevance of the shape and size of the ambiguity search space is emphasized. Also diagnostics are introduced which will be used in the sections following to characterize the quality of the ambiguities. In Sect. 4 we commence our study of the ambiguity search space. In order to give a qualitative description of the various phenomena, emphasis is given to analytical rather than numerical results.

It will be clear that with the ionospheric variance matrix, one has the flexibility to describe the shortbaseline case (ionosphere fixed), the long-baseline case (ionosphere floated), as well as all intermediate cases. The same holds true for the ambiguity variance matrices and the corresponding search spaces. We will use the following notation for the three different types of ambiguity variance matrix. When the ionosphere is fixed, it will be denoted as $Q_{\hat{a} \mid I}$. When the ionosphere is floated, it will be denoted as $Q_{\hat{a}}$, and when the ionosphere is weighted, it will be denoted as $Q_{\hat{a}}(I)$. The ambiguities have their best precision when the ionosphere is assumed known, and the poorest precision when the ionosphere is assumed completely unknown. Thus

$Q_{\hat{a} \mid I} \leq Q_{\hat{a}}(I) \leq Q_{\hat{a}}$

The variance matrix $Q_{\hat{a} \mid I}$ is the topic of Sect. 4 and the variance matrix $Q_{\hat{a}}$ the topic of Sect. 5. The results of these two sections thus set the bounds for the ambiguity variance matrix $Q_{\hat{a}}(I)$.

In Sect. 6 we commence with the a priori weighting of the ionosphere and show how it effects the a posteriori precision of the ionospheric estimators. The impact both of the spatial correlation and the time correlation is considered. Based on the results of this section, we are able in Sect. 7 to give an exact description of the interpolatory character of the ambiguity variance matrix $Q_{\hat{a}}(I)$. Also its dependency on the spatial correlation and on the time correlation is analysed. In this section we also show how the size of the ambiguity search space blows up or shrinks in as function of the a priori ionospheric weights. Finally, a summary of the main results is given in Sect. 8 .

\section{The geometry-free model}

In this section we will define the model that forms the basis of our analysis. Since the observation equations of the model are parametrized in terms of the DD receiversatellite ranges, the model dispenses with the relative receiver-satellite geometry. The model will therefore be referred to as the geometry-free single-baseline model. For integer ambiguity estimation this model is the simplest one can think of. It allows one to use the code data almost directly in combination with the phase data, to determine the integer ambiguities. See e.g. Hatch (1982), Wübbena (1988), Euler and Goad (1990), Dedes and Goad (1994) or Teunissen (1996). In the latter study, the geometry-free model was analysed with regard to its potential for ambiguity decorrelation.

The DD phase and code observation equations of the geometry-free model are given for a single epoch $i$, as

$$
\begin{aligned}
& \phi_{1}(i)=\rho(i)-\mu_{1} I(i)+\lambda_{1} a_{1} \\
& \phi_{2}(i)=\rho(i)-\mu_{2} I(i)+\lambda_{2} a_{2} \\
& p_{1}(i)=\rho(i)+\mu_{1} I(i) \\
& p_{2}(i)=\rho(i)+\mu_{2} I(i)
\end{aligned}
$$

where $\phi_{1}$ and $\phi_{2}$ are the DD phase observables on $L_{1}$ and $L_{2} ; p_{1}$ and $p_{2}$ are the DD code observables on $L_{1}$ and $L_{2} ; \rho$ is the DD form of the unknown receiver-satellite range; $I$ is the DD form of the unknown ionospheric delay, and $a_{1}$ and $a_{2}$ are the unknown integer ambiguities. The known wavelengths are denoted as $\lambda_{1}, \lambda_{2}$. Since the ionospheric delay is to a first order inversely proportional to the square of the frequency, we have to the same degree of approximation

$\mu_{1}=\frac{\lambda_{1}}{\lambda_{2}}=\frac{60}{77}, \quad \mu_{2}=\frac{\lambda_{2}}{\lambda_{1}}=\frac{77}{60}$

It will be assumed that the GPS observables are neither correlated between channels nor correlated in time. For $k$ epochs $(i=1, \ldots, k)$, their variance matrix is assumed to be given as

$I_{k} \otimes \operatorname{diag}\left(\sigma_{\phi_{1}}^{2}, \sigma_{\phi_{2}}^{2}, \sigma_{p_{1}}^{2}, \sigma_{p_{2}}^{2}\right)$

where $I_{k}$ is the unit matrix of order $k$ and $\otimes$ denotes the Kronecker product. Note that we allow the variances of the $L_{1}$ observables to be different from the $L_{2}$ observables. In our main results one will therefore still be free to set the level of precision of the observables. This also has the additional advantage that we can consider subsets of the observables. For instance, instead of having code data on both frequencies, one may also consider having code data available on a single frequency only. Or, when some a priori knowledge is assumed to be available on the ionosphere, one can consider the single-frequency case, instead of the dual-frequency case.

In addition to the observation equations in Eq. (1), we also consider for the $k$ epochs the ionospheric observation equations

$I_{p}(i)=I(i)$

$i=1, \ldots, k$, where $I_{p}$ is the ionospheric pseudo-observable. Its sample value can be taken from an externally provided ionospheric model, see e.g. Georgiadou (1994), Wild (1994), Wanninger (1995). In some applications it may even suffice to take zero as sample value. Our presumed knowledge of the a priori uncertainty in the ionospheric delay is modelled through an appropriate variance covariance matrix, see e.g. Delikaraoglou (1989), Goad (1990), Wild and Beutler (1991), Schaer (1994). In our case this matrix is of order $k$ and we will factor it as

$s_{I}^{2} R_{I}$

where $s_{I}^{2}$ is the variance factor of unit weight and $R_{I}$ is the cofactor matrix of order $k$. These two components can each be assigned a specific meaning 
$s_{I}^{2}$ : spatial correlation

$R_{I}$ : time correlation

The a priori uncertainty in the absolute level of the DD ionosphere is modelled through $s_{I}^{2}$ and the a priori uncertainty in the relative behaviour of $I(i)$ as function of time, is modelled through the correlation matrix $R_{I}$.

The value of $s_{I}^{2}$ depends in a large part on the interstation distance between the two receivers. Since the ionosphere decorrelates as function of the interstation distance, $s_{I}^{2}$ is at its maximum for baselines where the ionosphere is fully decorrelated and it gets smaller the shorter the baselines become. For sufficiently short baselines, it can be taken equal to zero. A proposal on how to describe $s_{I}^{2}$ as function of the interstation distance can be found in Bock (1996).

The correlation matrix $R_{I}$ depends on the sampling rate in relation to the correlation length of the ionosphere. It equals the unit matrix of order $k$ in case the sampling rate is so low that the time correlation of the ionosphere can be neglected. For a higher sampling rate though, $R_{I}$ will become a full matrix.

Based on the principle of least squares, the preceding model of observation equations can either be solved in batch form or recursive form. The recursive form (e.g. Kalman filter) may be advantageous when the number of samples $k$ is large. However, in order to be able to formulate the recursion, additional assumptions need to be made about the structure of the correlation matrix $R_{I}$. For this purpose a useful model is one where the ionospheric noise is assumed to be autoregressive. Then

$R_{I}=\left[\rho_{t}^{|i-j|}\right]$

where $i$ and $j$ refer to the column and row of the matrix and where $\rho_{t}$ can be taken as

$\rho_{t}=e^{-T / t}$

with the sampling interval $T$ and the correlation length $t$. The inverse of this correlation matrix has the upper triangular decomposition

$R_{I}^{-1}=L^{T} D L$

with

$D^{-1}=\operatorname{diag}\left[1,\left(1-\rho_{t}^{2}\right), \ldots,\left(1-\rho_{t}^{2}\right)\right]$

and

$L=\left[\begin{array}{cccc}1 & & & \\ -\rho_{t} & 1 & & \\ & \ddots & \ddots & \\ & & -\rho_{t} & 1\end{array}\right]$

It is the bidiagonal structure of $L$ which makes it possible to formulate the least-squares solution in recursive form. For the moment however, we will not make any specific assumptions concerning the structure of $R_{I}$. This implies that the results we will obtain are valid for batch solutions as well as for recursive solutions. We refer to the model as ionosphere fixed when
$s_{I}^{2}=0$, as ionosphere floated when $s_{I}^{2}=\infty$ and as ionosphere weighted when $0<s_{I}^{2}<\infty$.

\section{Shape and size of search space}

In this section we briefly describe the least-squares method of estimating the integer ambiguities and introduce some diagnostics for characterizing the geometry of the search space.

\subsection{Integer least squares}

When the principle of least-squares is used, the corresponding integer estimates of the ambiguities follow from solving the minimization problem

$\min _{a}(\hat{a}-a)^{T} Q_{\hat{a}}^{-1}(\hat{a}-a), \quad a \in Z^{2}$

where $\hat{a}$ is the real-valued least-squares estimate of the ambiguity vector and $Q_{\hat{a}}$ is its corresponding variance covariance matrix. This minimization is formulated for the case where the ionosphere is floated, but similar formulations hold of course for the two other cases as well.

When the method of the least-squares ambiguity decorrelation adjustment (LAMBDA) is used (Teunissen 1993), the solution steps to solve the integer leastsquares problem are, briefly, as follows. First the global search space $Z^{2}$ is replaced by a local one, the so-called ambiguity search space. It reads

$(\hat{a}-a)^{T} Q_{\hat{a}}^{-1}(\hat{a}-a) \leq \chi^{2}$

This is an elliptic region, centred at $\hat{a}$. Its shape is governed by the ambiguity variance matrix $Q_{\hat{a}}$ and its size can be controlled by choosing an appropriate value for the positive constant $\chi^{2}$. Both the shape and size of the search space have an important impact on the performance of the search. The search is hindered by a too-elongated search space of which the principal axes fail to be aligned with the grid axes. Also too large a value of $\chi^{2}$ should be avoided, since in that case the search space will contain an abundance of unnecessary grid points.

In order to come up with an appropriate size and shape of the search space, the original DD ambiguities are transformed to new ambiguities. These new ambiguities have the property that they are far less correlated than the original DD ambiguities and also more precise. If we denote the ambiguity transformation as $Z^{T}$, then

$z=Z^{T} a, \quad \hat{z}=Z^{T} \hat{a}, \quad Q_{\hat{z}}=Z^{T} Q_{\hat{a}} Z$

and the original DD search space Eq. (8) transforms accordingly to

$(\hat{z}-z)^{T} Q_{\hat{z}}^{-1}(\hat{z}-z) \leq \chi^{2}$

The decorrelating ambiguity transformation $Z^{T}$ is area preserving and it is constructed from a sequence of 
Gaussian transformations, which themselves are integer approximations of conditional least-squares transformations.

The preceding transformation takes care of the shape of the search space. In order to take care of the size of the search space, an appropriate value for $\chi^{2}$ needs to be chosen; one which is neither too large nor too small. It is based on applying a simple integer rounding scheme to the entries of $\hat{z}$. The integer vector so obtained is then substituted for $z$ in Eq. (10) and $\chi^{2}$ is then taken equal to the value of the quadratic form. This then ensures that the search space will contain the solution sought and also that it is likely to contain not too many more grid points. This last property is due to low correlation and high precision of the transformed ambiguities. Numerical examples showing how well this approach works are given in Teunissen et al. (1996) for a variety of different measurement scenarios.

Once we have obtained an appropriate shape and size of the search space, one can commence with the search. It is based on a conditional least-squares adjustment and can be briefly described as follows. Along the first, say horizontal grid axis, the search space defines an interval for the first ambiguity $z_{1}$. Once a candidate integer value has been chosen for $z_{1}$ from this interval, the search space defines another interval from which candidate integer values for the second ambiguity $z_{2}$ can be chosen. This second interval has the vertical width of the search space that corresponds with the chosen value of $z_{1}$. All grid points of the search space can now be collected as follows. By letting $z_{1}$ range through all integer values of the first interval, one collects for each value of $z_{1}$, those integers $z_{2}$ that lie in the second interval. They are the candidate integer values for the second ambiguity. In this way all integer pairs $\left(z_{1}, z_{2}\right)$ are collected that satisfy Eq. (10). The required integer least-squares solution is then given by that pair which returns the smallest value for the quadratic form. More details on the described procedure can be found in Teunissen (1995). Implementation aspects of the method are described in de Jonge and Tiberius (1996).

\subsection{Some ambiguity diagnostics}

In order to infer the shape and size of the ambiguity search space, we will make use of the following diagnostics: area $S$, correlation $\rho$ and elongation $e$. The area of the ambiguity search space is defined as

$S=\pi \chi^{2} \sqrt{\left|Q_{\hat{a}}\right|}$

where $\left|Q_{\hat{a}}\right|$ is the determinant of the ambiguity variance matrix. The area gives an indication of the number of grid points inside the search space.

Apart from the statistical interpretation of the correlation coefficient, it can also be given a geometrical interpretation. It is related to the area of the search space as

$$
\sqrt{1-\rho^{2}}=\frac{S}{\pi \chi^{2} \sigma_{a_{1}} \sigma_{a_{2}}}
$$

Note that $4 \chi^{2} \sigma_{a_{1}} \sigma_{a_{2}}$ equals the area of the box that encloses the search space and which has its sides parallel to the grid axes. This area is closest to $S$, when the ambiguities are fully decorrelated. However, the area of the search space is far smaller than the area of the box, when the correlation coefficient is close to 1 .

The elongation $e$ measures the outstretchedness of the search space. It equals the square root of the condition number of the ambiguity variance matrix. Thus it equals the ratio of the lengths of the major and minor principal axes of the search space. The area, the correlation and the elongation can nicely be connected to the precision of the ambiguities, if we consider their arithmetic and geometric mean. We have

$\left\{\begin{array}{l}\frac{1}{2}\left(\sigma_{a_{1}}^{2}+\sigma_{a_{2}}^{2}\right)=\frac{S}{2 \pi \pi^{2}}\left(e+e^{-1}\right) \\ \left(\sigma_{a_{1}}^{2} \sigma_{a_{2}}^{2}\right)^{1 / 2}=\frac{S}{\pi \chi^{2}}\left(1-\rho^{2}\right)^{-1 / 2}\end{array}\right.$

The first equation shows how the arithmetic mean of the ambiguity variances depends on the area and on the elongation of the search space. The second equation shows how the geometric mean of the ambiguity variances depends on the area and on the correlation coefficient. These two equations show that the ambiguities can be of a very poor precision, while the area of their search space is still small. In that case the ambiguities are highly correlated and their search space is very elongated. As we will see in the sections following, this is precisely what happens with the DD ambiguities. The preceding also shows how the precision of the ambiguities improves when the elongation and correlation are reduced. This is what happens when the areapreserving ambiguity transformation $Z^{T}$ of Eq. (9) is used.

The aforementioned diagnostics will be used to characterize and compare the geometries of the ambiguity search spaces when the ionosphere is either fixed, floated or weighted. In the following two sections we will first consider the two extreme cases, namely the ionosphere-fixed case and the ionosphere-float case.

\section{The ionosphere-fixed solution}

In this section we analyse the ambiguity search space for the case where the ionosphere is fixed. Hence, we restrict our attention to the observation equations of Eq. (1) and assume $I(i)$ to be identically zero for all $i$.

Let us first consider a single epoch. For a single epoch we have a redundancy of 1 . It stems from the code observable on the second frequency. Due to the structure of the observation equations, the single-epoch leastsquares estimate of the DD range is given as the weighted mean of the two code observables. The singleepoch least-squares estimates of the two ambiguities follow then from subtracting this weighted mean from the carrier phases followed by a division with the appropriate wavelength. Since the ambiguities are assumed to be constant in time and the DD ranges are assumed to be unconnected in time, the least-squares estimates of the two ambiguities based on $k$ epochs, simply follow as 
the time-averages of their single-epoch solutions. We thus have

$\hat{a}_{1 \mid I}=\frac{1}{\lambda_{1} k} \sum_{i=1}^{k}\left[\phi_{1}(i)-p_{w}(i)\right]$

$\hat{a}_{2 \mid I}=\frac{1}{\lambda_{2} k} \sum_{i=1}^{k}\left[\phi_{2}(i)-p_{w}(i)\right]$

with the weighted means

$p_{w}(i)=\frac{\sigma_{p_{2}}^{2} p_{1}(i)+\sigma_{p_{1}}^{2} p_{2}(i)}{\sigma_{p_{1}}^{2}+\sigma_{p_{2}}^{2}}$

An application of the error propagation law gives the variance matrix of the ambiguities as

$Q_{\hat{a}}=\frac{\sigma_{p_{w}}^{2}}{\lambda_{1} \lambda_{2} k}\left[\begin{array}{cc}\mu_{2}\left(1+\frac{\sigma_{\phi_{1}}^{2}}{\sigma_{p_{w}}^{2}}\right) & 1 \\ 1 & \mu_{1}\left(1+\frac{\sigma_{\phi_{2}}^{2}}{\sigma_{p_{w}}^{2}}\right)\end{array}\right]$

Note that this matrix can be written as the sum of a diagonal full-rank matrix and a rank-1 matrix. The entries of the diagonal matrix are very small due to the very high precision of the carrier-phase data. The entries of the rank-1 matrix, however, are large. This is due to the relatively poor precision of the code data. This type of decomposition, where the ambiguity variance matrix can be written as the sum of a full-rank matrix with small entries and a matrix of less than full rank with large entries, is very typical of GPS when the ionosphere is assumed fixed (Teunissen 1993).

\subsection{Area of search space}

It follows from the diagonal entries of Eq. (15) that the precision of the individual DD ambiguities is rather poor, unless $k$ is sufficiently large. In fact their precision is not too different from what one would get in the single-frequency case. In the $L_{1}$-only case, the variance of the $L_{1}$ ambiguity is given as

$\sigma_{a_{1 \mid I}}^{2}=\frac{\sigma_{\phi_{1}}^{2}}{\lambda_{1}^{2} k}\left(1+\sigma_{p_{1}}^{2} / \sigma_{\phi_{1}}^{2}\right)$

whereas in the dual-frequency case, it is given as

$\sigma_{a_{1 \mid I}}^{2}=\frac{\sigma_{\phi_{1}}^{2}}{\lambda_{1}^{2} k}\left(1+\sigma_{p_{w}}^{2} / \sigma_{\phi_{1}}^{2}\right)$

From this one should, however, not too hastily conclude that ambiguity validation in the dual-frequency case will be as problematic as it is in the single-frequency case. This becomes clear if we consider the area of the dualfrequency ambiguity search space. For the area, it suffices to concentrate on the determinant of the ambiguity variance matrix. It follows from Eq. (15) as
$\left|Q_{\hat{a} \mid I}\right|=\frac{\sigma_{\phi_{1}}^{2} \sigma_{\phi_{2}}^{2}}{\lambda_{1}^{2} \lambda_{2}^{2} k^{2}}\left(1+\sigma_{p_{w}}^{2} / \sigma_{\phi_{w}}^{2}\right)$

where $\sigma_{\phi_{w}}^{2}$ is the variance of the weighted mean of the $L_{1}$ and $L_{2}{ }$ carrier phases. This determinant expression shows two things. First it shows, as is the case with the individual variances already seen, that the area blows up the smaller the phase-code variance ratio gets. Thus as one might expect, the area gets larger the poorer the precision of the code data becomes. But the result also shows that the area, when squared, is only linearly related to the inverse of the phase-code variance ratio, whereas in the single-frequency case it would be quadratically related to it. Note that in order to compare the determinant with the variances, one should square the latter or take the square root of the former. Hence the area can still be small, despite the poor precision of the individual ambiguities. This shows the beneficial role which is played by having data on a second frequency available.

\subsection{Ambiguity correlation}

In order to explain the relatively small area of the search space, we consider the ambiguity correlation coefficient. In the remaining part of this section we will assume for reasons of simplicity that the two phase variances are equal and that the two code variances are equal.

The ambiguity correlation coefficient $\rho_{a \mid I}$ follows from Eq. (15) as

$\rho_{a \mid I}=\left(1+2 \sigma_{\phi}^{2} / \sigma_{p}^{2}\right)^{-1}$

Hence the ambiguity correlation coefficient is independent of the number of samples used. It is uniquely determined by the phase-code variance ratio. Since in practice this ratio is very small indeed (e.g. $10^{-4}$ ), it follows that the two ambiguities are extremely highly correlated. And it is precisely due to this high correlation, that one will be able to execute a successful validation, despite the fact that the two individual DD ambiguities themselves are of a poor precision. Thus one should never consider the ambiguities on an individual basis. Instead one should take all available information into account and thus include the correlation as well, when estimating and validating the integer ambiguities.

\subsection{Orientation and elongation}

We first consider the orientation of the search space. It follows from Eq. (15) as

$\theta_{a \mid I}=\frac{1}{2} \arctan \left(\frac{2}{\left(\mu_{2}-\mu_{1}\right)\left(1+2 \sigma_{\phi}^{2} / \sigma_{p}^{2}\right)}\right)$

where $\theta_{a \mid I}$ is the angle between the major axis of the search space and the grid axis of the first ambiguity. As 
it was the case with the correlation coefficient, the orientation is uniquely determined by the phase-code variance ratio. It equals approximately $38^{\circ}$ for a phasecode variance ratio of $10^{-4}$.

The elongation equals the square root of the condition number of the ambiguity variance matrix and it follows from Eq. (15) as

$e_{a \mid I}=\left[\left(1+\sqrt{1-r_{a \mid I}^{2}}\right) /\left(1-\sqrt{1-r_{a \mid I}^{2}}\right)\right]^{1 / 2}$

with

$\frac{2}{r_{a \mid I}}=\frac{1}{2}\left(\mu_{1}+\mu_{2}\right)\left[\left(1+\sigma_{p}^{2} / \sigma_{\phi}^{2}\right)^{-1 / 2}+\left(1+\sigma_{p}^{2} / \sigma_{\phi}^{2}\right)^{+1 / 2}\right]$

Note that $\frac{2}{r_{a \mid I}}=e_{a \mid I}+\frac{1}{e_{a \mid l}}$. This shows that $\frac{2}{r_{a \mid I}}$ itself is a good approximation for the elongation, when the elongation is large. Since $r_{a \mid I}$ is small when the phasecode variance ratio is small, it follows that the search consider the ambiguity variance matrix and describe the size and shape of the ambiguity search space.

\subsection{Ionospheric precision}

We will discriminate between two cases. The first case considers the precision of the ionospheric delays when the ambiguities are fixed, while in the second case we consider the ambiguities to be floated. Thus in the first case we have the variance matrix $Q_{\hat{I} \mid a}$, while in the second, the variance matrix $Q_{\hat{I}}$.

5.1.1 Ambiguities fixed It follows from the observation equations of Eq. (1) that for a single epoch, the precision with which the ionospheric delay $I(i)$ can be estimated when the two ambiguities are assumed known, is given as

$$
\sigma_{I \mid a}^{2}=\frac{\left(\sigma_{\phi_{1}}^{2}+\sigma_{\phi_{2}}^{2}\right) \sigma_{p_{1}}^{2} \sigma_{p_{2}}^{2}+\left(\sigma_{p_{1}}^{2}+\sigma_{p_{2}}^{2}\right) \sigma_{\phi_{1}}^{2} \sigma_{\phi_{2}}^{2}}{\left(\sigma_{\phi_{1}}^{2}+\sigma_{p_{1}}^{2}\right)\left(\sigma_{\phi_{2}}^{2}+\sigma_{p_{2}}^{2}\right)\left(\mu_{1}-\mu_{2}\right)^{2}+4\left(\sigma_{\phi_{2}}^{2} \mu_{1}+\sigma_{\phi_{1}}^{2} \mu_{2}\right)\left(\sigma_{p_{2}}^{2} \mu_{1}+\sigma_{p_{1}}^{2} \mu_{2}\right)}
$$

space is indeed very elongated. The elongation equals 103 for a phase-code variance ratio of $10^{-4}$

From the preceding we can conclude that it is the very high precision of the phase data relative to the rather poor precision of the code data that causes the ambiguities to be extremely correlated and their search space to be very elongated. The ambiguities will become less correlated and their search space will become less elongated when the precision of the code data improves with respect to the precision of the phase data. For the two extreme cases that $\sigma_{\phi}^{2}=\sigma_{p}^{2}$ or $\sigma_{\phi}^{2}=0$, the correlation, orientation and elongation are bounded as

$$
\begin{aligned}
\frac{1}{3} & \leq \rho_{a \mid I} \leq 1 \\
26.5^{\circ} & \leq \theta_{a \mid I} \leq 37.9^{\circ} \\
1.5 & \leq e_{a \mid I} \leq \infty
\end{aligned}
$$

These results only hold true, of course, when the ionospheric delays can be assumed absent from the model. This is thus the situation one will encounter for sufficiently short baselines. The situation will change however, when the ionospheric delays become part of the set of unknown parameters. In order to infer the impact of the presence of the ionospheric delays on the ambiguity search space, we will now consider the other extreme, the ionosphere-float solution.

\section{The ionosphere-float solution}

Since the ionospheric delays are now part of the set of unknown parameters, we will first consider the precision with which they can be estimated. After that, we will
If we assume the two phase variances to be equal and the two code variances to be equal, this expression simplifies to

$$
\sigma_{I \mid a}^{2}=\frac{2 \sigma_{\phi}^{2} /\left(1+\sigma_{\phi}^{2} / \sigma_{p}^{2}\right)}{\left(\mu_{1}-\mu_{2}\right)^{2}+4\left(\sigma_{\phi}^{2} / \sigma_{p}^{2}\right)\left(\mu_{1}+\mu_{2}\right)^{2} /\left(1+\sigma_{\phi}^{2} / \sigma_{p}^{2}\right)^{2}}
$$

Since the phase-code variance ratio is very small, we have the approximation

$\sigma_{I \mid a}^{2} \simeq \frac{2 \sigma_{\phi}^{2}}{\left(\mu_{1}-\mu_{2}\right)^{2}}$

This in fact is the precision with which the ionosphere can be estimated when the code data would be absent, but the ambiguities fixed. It shows that a very precise monitoring of the ionosphere is possible once one has been able successfully to validate the integer ambiguities. For an undifferenced phase standard deviation of $3 \mathrm{~mm}$, the ionospheric standard deviation equals $\sigma_{I \mid a} \simeq 1.7 \mathrm{~cm}$.

Since the observation equations are unconnected in time when the ambiguities are assumed known, it follows that the ionospheric variance matrix based on $k$ epochs is simply given by the scaled unit matrix

$Q_{\hat{I} \mid a}=\sigma_{I \mid a}^{2} I_{k}$

Thus when the ambiguities are fixed, the ionospheric estimator has a constant variance and is uncorrelated in time. Furthermore, since its variance is dominated by the precision of the phase observables, it has a very good precision. 
5.1.2 Ambiguities float The two ionospheric variance matrices $Q_{\hat{I}}$ and $Q_{\hat{I} \mid a}$ are related as

$Q_{\hat{I}}=Q_{\hat{I} \mid a}+Q_{\hat{I} \hat{a}} Q_{\hat{a}}^{-1} Q_{\hat{a} \hat{I}}$

This equation directly follows from the definition of a conditional variance matrix. We will first consider the precision with which time-differences of the ionosphere can be estimated. It follows from the structure of Eq. (1) that the covariance between $\hat{a}$ and $\hat{I}(i)$ is constant in time. This implies that for every full-rank matrix $D$ of order $k \times(k-1)$, with a range space $R(D) \perp R\left(e_{k}\right)$, where $e_{k}=(1, \ldots, 1)^{T}$, we have $D^{T} e_{k}=0$ and $D^{T} Q_{\hat{I} \hat{a}}=0$. This, combined with Eqs. (23) and (24); shows that

$D^{T} Q_{\hat{I}} D=D^{T} Q_{\hat{I} \mid a} D=\sigma_{I \mid a}^{2} D^{T} D$

$D^{T} Q_{\hat{I}} e_{k}=D^{T} Q_{\hat{I} \mid a} e_{k}=0$

This in turn shows that the ionospheric time differences are invariant to ambiguity fixing. Hence they can be estimated with a high precision even when the ionosphere is floated. This situation changes drastically however, when we consider instead of the relative behaviour of the ionosphere over time, its absolute level. It follows from Eq. (1) that for a single epoch only the code data contribute to the determination of $I(i)$. The precision with which the ionospheric delay can be estimated based on one single epoch, is given as

$\sigma_{I}^{2}=\frac{\sigma_{p_{1}}^{2}+\sigma_{p_{2}}^{2}}{\left(\mu_{2}-\mu_{1}\right)^{2}}$

Compare this with Eq. (22). With an undifferenced code standard deviation of $30 \mathrm{~cm}$, we have $\sigma_{I}=1.7 \mathrm{~m}$. Since the phase data not only fail to contribute to a singleepoch solution, but also to the sum of the ionospheric delays, we have

$e_{k}^{T} Q_{\hat{I}} e_{k}=k \sigma_{I}^{2}$

This result combined with Eq. (25) allows us to determine $Q_{\hat{I}}$. Since matrix $\left(D, e_{k}\right)$ is square and invertible, it follows from Eqs. 25 and 27 that $Q_{\hat{I}}=\sigma_{I \mid a}^{2} D\left(D^{T} D\right)^{-1} D^{T}+\sigma_{I}^{2} e_{k}\left(e_{k}^{T} e_{k}\right)^{-1} e_{k}^{T}$, or, since the projector $D\left(D^{T} D\right)^{-1} D^{T}$ can also be expressed as $I_{k}-e_{k}\left(e_{k}^{T} e_{k}\right)^{-1} e_{k}^{T}$, that

$Q_{\hat{I}}=\sigma_{I \mid a}^{2} I_{k}+\frac{1}{k}\left(\sigma_{I}^{2}-\sigma_{I \mid a}^{2}\right) e_{k} e_{k}^{T}$

Thus the ionospheric variance matrix in the case where the ionosphere is floated, differs from its counterpart when the ambiguities are fixed by a rank-1 matrix of which the entries are dominated by the poor precision of the code data.

\subsection{Ambiguity precision}

We will now consider the ambiguity variance matrix and the size and shape of the ambiguity search space. With the ionospheric delay included, the four equalities of Eq. (1) fail to be redundant. Hence, a single-epoch solution simply follows from inverting these four equations, and the least-squares ambiguity solution based on $k$ epochs then equals its time average. Thus for $k$ epochs, we have

$$
\begin{aligned}
& \hat{a}_{1}=\frac{1}{\lambda_{1} k} \sum_{i=1}^{k}\left[\phi_{1}(i)-v_{3}\left(p_{1}(i)-v_{1} p_{2}(i)\right)\right] \\
& \hat{a}_{2}=\frac{1}{\lambda_{2} k} \sum_{i=1}^{k}\left[\phi_{2}(i)-v_{3}\left(v_{2} p_{1}(i)-p_{2}(i)\right)\right]
\end{aligned}
$$

with

$$
\begin{aligned}
& v_{1}=\frac{2 \mu_{1}}{\mu_{1}+\mu_{2}} \simeq 0.76, \quad v_{2}=\frac{2 \mu_{2}}{\mu_{1}+\mu_{2}} \simeq 1.24, \\
& v_{3}=\frac{\mu_{2}+\mu_{1}}{\mu_{2}-\mu_{1}} \simeq 4.09
\end{aligned}
$$

Application of the error propagation law gives the ambiguity variance matrix as

$$
\begin{gathered}
Q_{\hat{a}}=\frac{1}{\lambda_{1} \lambda_{2} k}\left[\begin{array}{cc}
\mu_{2} \sigma_{\phi_{1}}^{2} & 0 \\
0 & \mu_{1} \sigma_{\phi_{2}}^{2}
\end{array}\right]+\frac{v_{3}^{2}}{\lambda_{1} \lambda_{2} k} \\
{\left[\begin{array}{cc}
\mu_{2}\left(\sigma_{p_{1}}^{2}+v_{1}^{2} \sigma_{p_{2}}^{2}\right) & v_{2} \sigma_{p_{1}}^{2}+v_{1} \sigma_{p_{2}}^{2} \\
v_{2} \sigma_{p_{1}}^{2}+v_{1} \sigma_{p_{2}}^{2} & \mu_{1}\left(v_{2}^{2} \sigma_{p_{1}}^{2}+\sigma_{p_{2}}^{2}\right)
\end{array}\right]}
\end{gathered}
$$

Note that the ambiguity variance matrix now remains of full rank when the phase variances are set to zero. A comparison with the ambiguity variance matrix of the ionosphere-fixed solution shows, if we neglect the very small phase-code variance ratio and assume equal variances for the phase data and equal variances for the code data, that the precision of the individual ambiguities is enlarged by a factor of

$\frac{\sigma_{a_{1}}}{\sigma_{a_{1 \mid I}}} \simeq v_{3} \sqrt{2\left(1+v_{1}^{2}\right)} \simeq 7.3$

5.2.1 Area of search space To show by how much the area of the search space is enlarged, we need the determinant of $Q_{\hat{a}}$. We can now use the results of the previous subsection to express $\left|Q_{\hat{a}}\right|$ in terms of $\left|Q_{\hat{a} \mid I}\right|$. It follows from Eq. (24) that $Q_{\hat{I}}^{-1} Q_{\hat{I} \mid a}=I_{k}-Q_{\hat{I}}^{-1} Q_{\hat{I} \hat{a}} Q_{\hat{a}}^{-1} Q_{\hat{a} \hat{I}}$. Similarly we have $Q_{\hat{a}}^{-1} Q_{\hat{a} \mid I}=I_{2}-Q_{\hat{a}}^{-1} Q_{\hat{a} \hat{I}} Q_{\hat{I}}^{-1} Q_{\hat{I} \hat{a}}$. Since for any two matrices $A$ and $B,|I-A B|=|I-B A|$, it follows that

$\left|Q_{\hat{a}}\right|\left|Q_{\hat{I} \mid a}\right|=\left|Q_{\hat{I}}\right|\left|Q_{\hat{a} \mid I}\right|$

The two determinants $\left|Q_{\hat{I} \mid a}\right|$ and $\left|Q_{\hat{I}}\right|$ follow from Eqs. (23) and (28) as

$$
\left|Q_{\hat{I} \mid a}\right|=\left(\sigma_{I \mid a}^{2}\right)^{k}, \quad\left|Q_{\hat{I}}\right|=\left(\sigma_{I \mid a}^{2}\right)^{k-1} \sigma_{I}^{2}
$$

Hence, the determinant of the ionosphere-float ambiguity variance matrix is given by the compact expression

$\left|Q_{\hat{a}}\right|=\left|Q_{\hat{a} \mid I}\right| \frac{\sigma_{I}^{2}}{\sigma_{I \mid a}^{2}}$ 
Since $\sigma_{I}^{2}$ is determined by the code variance and $\sigma_{I \mid a}^{2}$ predominantly by the phase variance, it follows that the multiplier by which the area gets blown up can be approximated as

$\frac{\left|Q_{\hat{a}}\right|}{\left|Q_{\hat{a} \mid I}\right|} \simeq \frac{\sigma_{p}^{2}}{\sigma_{\phi}^{2}}$

Thus the ratio of the two areas is approximately inversely proportional to the square root of the phasecode variance ratio. For a phase-code variance ratio of $10^{-4}$, the area of the ionosphere-float search space will be one hundred times larger than the area of the ionsphere-fixed search space. This shows that one will need quite some more samples in order to be able successfully to validate the integer ambiguities.

5.2.2 Ambiguity correlation In the remaining part of this section we will assume the two phase variances to be equal and the two code variances to be equal. The correlation coefficient follows from Eq. (30) as

$\rho_{a}=2 / \sqrt{\left(1+v_{1}^{2}+\sigma_{\phi}^{2} /\left(v_{3} \sigma_{p}\right)^{2}\right)\left(1+v_{2}^{2}+\sigma_{\phi}^{2} /\left(v_{3} \sigma_{p}\right)^{2}\right)}$

This shows that although the correlation is somewhat smaller than in the ionosphere-fixed case, it is still quite large. Hence one can still benefit considerably from the decorrelating ambiguity transformation $Z^{T}$.

5.2.3 Orientation and elongation The orientation of the major principle axis of the search space follows from Eq. (30) as

$\theta_{a}=\frac{1}{2} \arctan \frac{4 v_{3}^{2}}{\left(\mu_{2}-\mu_{1}\right)\left(1+\sigma_{\phi}^{2} / \sigma_{p}^{2}\right)}$

This shows, that when compared to the ionosphere-fixed case, the search space gets rotated counter-clockwise when the ionospheric delays are included as unknown parameters. The angle of rotation is, however, not that large, since $\theta_{a} \simeq 44.8^{\circ}$ for a phase-code variance ratio of $10^{-4}$. The elongation follows from Eq. (30) as

$e_{a}=\left[\left(1+\sqrt{1-r_{a}^{2}}\right) /\left(1-\sqrt{1-r_{a}^{2}}\right)\right]^{1 / 2}$

with

$\frac{2}{r_{a}}=\left(\mu_{1}+\mu_{2}\right) \frac{\sigma_{\phi}^{2} / \sigma_{p}^{2}+v_{3}^{2}\left(1+4 /\left(\mu_{1}+\mu_{2}\right)^{2}\right)}{\left[\left(1+\sigma_{\phi}^{2} / \sigma_{p}^{2}\right)^{2}+4 v_{3}^{2} \sigma_{\phi}^{2} / \sigma_{p}^{2}\right]^{1 / 2}}$

This shows that the search space is still quite elongated, although its elongation is less than in the ionospherefixed case.

From the preceding we can conclude that as in the ionosphere-fixed case, it is again the phase-code variance ratio which determines the shape and orientation of the search space. And again the ambiguities will be highly correlated and their search space very elongated. There are two important differences however. First, the area of the ionosphere-float search space is very much larger than its ionosphere-fixed counterpart. Secondly, the correlation, the orientation and the elongation are much less sensitive to changes in the phase-code variance ratio. For the two extreme cases that $\sigma_{\phi}^{2}=\sigma_{p}^{2}$ or $\sigma_{\phi}^{2}=0$, they are bounded as

$0.96977 \leq \rho_{a} \leq 0.99955$

$44.6^{\circ} \leq \theta_{a} \leq 44.8^{\circ}$

$8 \leq e_{a} \leq 67$

This shows that in particular the correlation and orientation only vary in a very small interval. Thus in the ionosphere-float case one cannot hope to decorrelate the ambiguities by much when the code data become more precise.

\section{The weighted ionosphere}

In the previous two sections we studied the characteristics of the two ambiguity variance matrices $Q_{\hat{a} \mid I}$ and $Q_{\hat{a}}$. In order to study their ionosphere-weighted counterpart $Q_{\hat{a}}(I)$, it turns out to be advantageous first to study the precision with which the weighted ionosphere itself can be estimated. This will therefore be the topic of the present section. We first derive an expression for the precision of the time-averaged ionosphere and then show how it is affected by the presence or absence of time correlation.

\subsection{The time-averaged ionosphere}

The unweighted ionospheric variance matrices $Q_{\hat{I} \mid a}$ and $Q_{\hat{I}}$ are related to their ionosphere-weighted counterparts as

$$
\begin{aligned}
Q_{\hat{I} \mid a}(I) & =\left[Q_{\hat{I} \mid a}^{-1}+s_{I}^{-2} R_{I}^{-1}\right]^{-1} \\
& =Q_{\hat{I} \mid a}-Q_{\hat{I} \mid a}\left[Q_{\hat{I} \mid a}+s_{I}^{2} R_{I}\right]^{-1} Q_{\hat{I} \mid a} \\
Q_{\hat{I}}(I) & =\left[Q_{\hat{I}}^{-1}+s_{I}^{-2} R_{I}^{-1}\right]^{-1} \\
& =Q_{\hat{I}}-Q_{\hat{I}}\left[Q_{\hat{I}}+s_{I}^{2} R_{I}\right]^{-1} Q_{\hat{I}}
\end{aligned}
$$

In the first case the ambiguities are fixed, while in the second they are floated. Matrix $s_{I}^{2} R_{I}$ is the a priori variance matrix of the ionospheric delays.

It follows from Eq. (37) that between the four ionospheric variance matrices, the following relation holds

$Q_{\hat{I}}(I)=\left[Q_{\hat{I} \mid a}^{-1}(I)-Q_{\hat{I} \mid a}^{-1}+Q_{\hat{I}}^{-1}\right]^{-1}$

This matrix relation is generally valid and does not depend on the particular structure of the model of observation equations. We will show however, that a similar relation, but now in scalar form, exists for the precision of the time-averaged ionosphere. This relation is valid due to the particular structure of the model of observation equations. 
For the four cases, we use the following notation for the variances of the time-averaged ionosphere

$$
\begin{array}{ll}
\sigma_{\bar{I} \mid a}^{2}=\frac{1}{k^{2}} e_{k}^{T} Q_{\hat{I} \mid a} e_{k} & \sigma_{\bar{I}}^{2}=\frac{1}{k^{2}} e_{k}^{T} Q_{\hat{I}} e_{k} \\
\sigma_{\bar{I} \mid a}^{2}(I)=\frac{1}{k^{2}} e_{k}^{T} Q_{\hat{I} \mid a}(I) e_{k} & \sigma_{\bar{I}}^{2}(I)=\frac{1}{k^{2}} e_{k}^{T} Q_{\hat{I}}(I) e_{k}
\end{array}
$$

As to their size, these variances are related as

$$
\sigma_{\bar{I} \mid a}^{2}(I) \leq \sigma_{\bar{I}}^{2}(I) \leq \sigma_{\bar{I}}^{2}, \quad \sigma_{\bar{I} \mid a}^{2} \leq \sigma_{\bar{I}}^{2}
$$

Which one of the two inequalities of $\sigma_{\bar{I}}^{2}(I) \leq \geq \sigma_{\bar{I} \mid a}^{2}$ is valid depends on the relative impact of $s_{I}^{2} R_{I}$.

In order to determine $\sigma_{\bar{I}}^{2}(I)$, we first substitute Eqs. 23 and 28 into Eq. (38). This gives

$$
\begin{aligned}
Q_{\hat{I}}(I) & =\left[Q_{\hat{I} \mid a}^{-1}(I)-\left(\sigma_{\bar{I} \mid a}^{-2}-\sigma_{\bar{I}}^{-2}\right) e_{k} e_{k}^{T}\right]^{-1} \\
& =Q_{\hat{I} \mid a}(I)+\frac{1}{k^{2}} \frac{Q_{\hat{I} \mid a}(I) e_{k} e_{k}^{T} Q_{\hat{I} \mid a}(I)}{\sigma_{\bar{I}}^{2} \sigma_{\bar{I} \mid a}^{2} /\left(\sigma_{\bar{I}}^{2}-\sigma_{\bar{I} \mid a}^{2}\right)-\sigma_{\bar{I} \mid a}^{2}(I)}
\end{aligned}
$$

From summing all its entries and dividing the sum by $k^{2}$, the required analogue to Eq. (38) follows as

$\sigma_{\bar{I}}^{2}(I)=\left[\sigma_{\bar{I} \mid a}^{-2}(I)-\sigma_{\bar{I} \mid a}^{-2}+\sigma_{\bar{I}}^{-2}\right]^{-1}$

Note that this result is still independent of the structure one might choose for the a priori ionospheric variance matrix $s_{I}^{2} R_{I}$. This result of course reduces to $\sigma_{\bar{I}}^{2}$ when the ionospheric weights are absent, and thus $\sigma_{\bar{I} \mid a}^{2}(I)=\sigma_{\bar{I} \mid a}^{2}$. But when the weights are such that $\sigma_{\bar{I} \mid a}^{2}(I)$ is sufficiently smaller than $\sigma_{\bar{I} \mid a}^{2}$, then $\sigma_{\bar{I}}^{2}$ can be neglected due to the high precision of the phase data relative to the precision of the code data, and we have the approximation

$\sigma_{\bar{I}}^{2}(I) \simeq \frac{\sigma_{\bar{I} \mid a}^{2} \sigma_{\bar{I} \mid a}^{2}(I)}{\sigma_{\bar{I} \mid a}^{2}-\sigma_{\bar{I} \mid a}^{2}(I)}$

The larger the spatial correlation is or the smaller the baselines are, the better the approximation becomes.

\subsection{The impact of time correlation}

In order to infer the impact of time correlation, we will first consider two extreme cases. In the first case we assume the time correlation to be absent $\left(\rho_{t}=0\right)$. This corresponds to the case of having such a low sampling rate that one can safely neglect the correlation of the ionosphere in time. In the second case, we assume the time correlation to be at its maximum $\left(\rho_{t}=1\right)$. Hence this corresponds to the use of a very high sampling rate. After having considered the two extreme cases, we will consider the case that $0<\rho_{t}<1$. For this case, we will assume the time correlation to be autoregressive.

6.2.1 Ionosphere with white noise $\left(\rho_{t}=0\right)$ If the time correlation may be assumed absent $\left(\rho_{t}=0\right)$, the correlation matrix becomes identical to the unit matrix of order $k$, $R_{I}=I_{k}$. The two variances of the time-averaged ionosphere, for the case the ambiguities are fixed and for the case where the ambiguities are floated, are then given as

$$
\left\{\begin{array}{c}
\sigma_{\bar{I}}^{2}\left(I, \rho_{t}=0\right)=\frac{1}{k} \frac{\sigma_{I \mid a}^{2} s_{I}^{2}}{\sigma_{I \mid a}^{2}+s_{I}^{2}} \\
\sigma_{\bar{I}}^{2}\left(I, \rho_{t}=0\right)=\frac{1}{k} \frac{\sigma_{I}^{2} s_{I}^{2}}{\sigma_{I}^{2}+s_{I}^{2}}
\end{array}\right.
$$

The first equation follows from substituting $R_{I}=I_{k}$ into Eq. (37), followed by taking the sum of all entries of $Q_{\hat{I} \mid a}(I)$ and dividing the sum by $k^{2}$. The second equation follows from substituting the first into Eq. (39).

Note that both variances are proportional to the inverse of the number of samples used. The first variance is predominantly governed by the phase variances, whereas the second variance is independent of the phase variances. The second variance depends on the code variances and on the variance factor $s_{I}^{2}$. Hence it will be large, unless a sufficient number of samples are taken, or, unless $s_{I}^{2}$ is small (large spatial correlation or short baselines). Due to the high precision of the phase data, the first variance is small even when $s_{I}^{2}$ is large. This shows the benefit of successful ambiguity fixing. Once the ambiguities are fixed, the degrading effect of having a low spatial correlation is then considerably reduced.

6.2.2 Ionosphere as random constant $\left(\rho_{t}=1\right)$ In case the time correlation is at its maximum, the correlation matrix reduces to the rank-1 matrix $R_{I}=e_{k} e_{k}^{T}$. The two variances of the time-averaged ionosphere are then given as

$$
\left\{\begin{array}{l}
\sigma_{\bar{I} \mid a}^{2}\left(I, \rho_{t}=1\right)=\frac{1}{k} \frac{\sigma_{I \mid a}^{2} k s_{I}^{2}}{\sigma_{I \mid a}^{2}+k s_{I}^{2}} \\
\sigma_{\bar{I}}^{2}\left(I, \rho_{t}=1\right)=\frac{1}{k} \frac{\sigma_{I}^{2} k s_{I}^{2}}{\sigma_{I}^{2}+k s_{I}^{2}}
\end{array}\right.
$$

The first equation follows from substituting $R_{I}=e_{k} e_{k}^{T}$ into Eq. (37), followed by taking the sum of all entries of $Q_{\hat{I} \mid a}(I)$ and dividing the sum by $k^{2}$. The second equation follows from substituting the first into Eq. (39).

Note that Eq. (41) can be obtained from Eq. (40) simply by replacing $s_{I}^{2}$ by $k s_{I}^{2}$. This shows that the presence of time correlation amplifies the presence of spatial decorrelation. Hence, by using a lower sampling rate, thus avoiding time correlation, one can reach the same precision using longer baselines. Or in other words, a high sampling rate virtually shortens the baseline.

6.2.3 Ionosphere with autoregressive noise In order to consider the case $0<\rho_{t}<1$, we assume the ionospheric noise to be autoregressive and thus that $R_{I}=\left[\rho_{t}^{|i-j|}\right]$. For this case however, it is not possible to obtain an exact and simple closed-form formula for $\sigma_{\bar{I} \mid a}^{2}(I)$.

To study the influence of the autoregressive noise, we will make use of the first order approximation

$$
\sigma_{\bar{I} \mid a}^{2}(I) \simeq \sigma_{\bar{I} \mid a}^{2}\left(I, \rho_{t}^{0}\right)+\frac{\mathrm{d}}{\mathrm{d} \rho_{t}}\left[\sigma_{\bar{I} \mid a}^{2}\left(I, \rho_{t}^{0}\right)\right]\left(\rho_{t}-\rho_{t}^{0}\right)
$$


and assume that the time correlation is either small $\left(\rho_{t}^{0}=0\right)$ or large $\left(\rho_{t}^{0}=1\right)$. This allows us then to make use of our previous results Eqs. 40 and 41.

In order to determine the derivative of $\sigma_{\bar{I} \mid a}^{2}(I)$, we make use of

$$
\begin{gathered}
\frac{\mathrm{d}}{\mathrm{d} \rho_{t}}\left[I_{k}+\alpha^{-1} R_{I}^{-1}\right]^{-1}=\frac{\mathrm{d}}{\mathrm{d} \rho_{t}}\left[\alpha R_{I}\left(I_{k}+\alpha R_{I}\right)^{-1}\right] \\
=\left[I_{k}+\alpha R_{I}\right]^{-1} \frac{\mathrm{d}}{\mathrm{d} \rho_{t}}\left[\alpha R_{I}\right]\left[I_{k}+\alpha R_{I}\right]^{-1}
\end{gathered}
$$

For $\rho_{t}^{0}=0$, this gives

$R_{I}=I_{k}, \quad\left(I_{k}+\alpha R_{I}\right)^{-1}=\frac{1}{1+\alpha} I_{k}$

$\frac{\mathrm{d}}{\mathrm{d} \rho_{t}}\left[R_{I}\right]=\left[\begin{array}{ccccc}0 & 1 & & & \\ 1 & 0 & 1 & & \\ & \ddots & \ddots & \ddots & \\ & & 1 & 0 & 1 \\ & & & 1 & 0\end{array}\right]$

and for $\rho_{t}^{0}=1$, we obtain in a similar way

$$
\begin{gathered}
R_{I}=e_{k} e_{k}^{T}, \quad\left(I_{k}+\alpha R_{I}\right)^{-1}=I_{k}-\frac{\alpha}{1+\alpha k} e_{k} e_{k}^{T} \\
\frac{\mathrm{d}}{\mathrm{d} \rho_{t}}\left[R_{I}\right]=\left[\begin{array}{cccc}
0 & 1 & \ldots & (k-1) \\
1 & 0 & \ldots & (k-2) \\
\vdots & \vdots & 0 & 1 \\
(k-1) & (k-2) & 1 & 0
\end{array}\right]
\end{gathered}
$$

Using both these results together with Eq. (42), gives

$$
e_{k}^{T} \frac{\mathrm{d}}{\mathrm{d} \rho_{t}}\left[I_{k}+\alpha^{-1} R_{I}^{-1}\right]^{-1} e_{k}=\left\{\begin{array}{cc}
\frac{2 \alpha(k-1)}{(1+\alpha)^{2}} & \left(\rho_{t}^{0}=0\right) \\
\frac{1}{3} \frac{\alpha k(k-1)(k+1)}{(1+\alpha k)^{2}} & \left(\rho_{t}^{0}=1\right)
\end{array}\right.
$$

Since $\quad \sigma_{\bar{I} \mid a}^{2}(I)=\frac{1}{k^{2}} \sigma_{I \mid a}^{2} e_{k}^{T}\left[I_{k}+\alpha^{-1} R_{I}^{-1}\right]^{-1} e_{k} \quad$ with $\quad \alpha=$ $s_{I}^{2} / \sigma_{I \mid a}^{2}$, we may now use Eq. (43) together with Eqs. 40 and 41 to obtain our first-order approximations. The first-order approximations of $\sigma_{\bar{I}}^{2}(I)$, are obtained in a similar way, but are based on a linearization of Eq. (39).

As a final result, we get for small time correlations

$$
\left\{\begin{array}{l}
\sigma_{\bar{I} \mid a}^{2}(I) \simeq \frac{1}{k} \frac{\sigma_{I \mid a}^{2} s_{I}^{2}}{\sigma_{I \mid a}^{2}+s_{I}^{2}}\left[1+2 \rho_{t}\left(1-\frac{1}{k}\right) \frac{\sigma_{I \mid a}^{2}}{\sigma_{I}^{2}+s_{I}^{2}}\right] \\
\sigma_{\bar{I}}^{2}(I) \simeq \frac{1}{k} \frac{\sigma_{I}^{2} s_{I}^{2}}{\sigma_{I}^{2}+s_{I}^{2}}\left[1+2 \rho_{t}\left(1-\frac{1}{k}\right) \frac{\sigma_{I}^{2}}{\sigma_{I}^{2}+s_{I}^{2}}\right]
\end{array}\right.
$$

and for large time correlations

$$
\left\{\begin{array}{l}
\sigma_{\bar{I} \mid a}^{2}(I) \simeq \frac{1}{k} \frac{\sigma_{I \mid a}^{2} k s_{I}^{2}}{\sigma_{I \mid a}^{2}+k s_{I}^{2}}\left[1-\frac{1}{3}\left(1-\rho_{t}\right)\left(k-\frac{1}{k}\right) \frac{\sigma_{I \mid a}^{2}}{\sigma_{I \mid a}^{2}+k s_{I}^{2}}\right] \\
\sigma_{\bar{I}}^{2}(I) \simeq \frac{1}{k} \frac{\sigma_{I}^{2} k s_{I}^{2}}{\sigma_{I}^{2}+k s_{I}^{2}}\left[1-\frac{1}{3}\left(1-\rho_{t}\right)\left(k-\frac{1}{k}\right) \frac{\sigma_{I}^{2}}{\sigma_{I}^{2}+k s_{I}^{2}}\right]
\end{array}\right.
$$

Note that it is only the code data and not the phase data which contribute to $\sigma_{\bar{I}}^{2}(I)$. After ambiguity fixing however, the contribution stems predominantly from the phase data. Also note from the expressions between square brackets that the impact of non-zero time correlation reaches a steady state for sufficiently large $k$ and thus will act as a constant scale factor. In the case of Eq. (44) the scale factor is smaller for $\sigma_{\bar{I} \mid a}^{2}(I)$ than it is for $\sigma_{\bar{I}}^{2}(I)$. This is due to the high precision of the phase data. In the case of Eq. (45), the situation is of course reversed. Finally note, that these results clearly show that the presence of time correlation degrades the precision with which the ionosphere can be estimated. In fact we have the ordering

$$
\begin{gathered}
\sigma_{\bar{I} \mid a}^{2}\left(I, \rho_{t}=0\right) \leq \sigma_{\bar{I} \mid a}^{2}(I) \leq \sigma_{\bar{I} \mid a}^{2}\left(I, \rho_{t}=1\right) \\
\sigma_{\bar{I}}^{2}\left(I, \rho_{t}=0\right) \leq \sigma_{\bar{I}}^{2}(I) \leq \sigma_{\bar{I}}^{2}\left(I, \rho_{t}=1\right)
\end{gathered}
$$

Thus for a fixed value of $k$, it is more advantageous to use a low sampling rate than a high sampling rate.

\section{Ionosphere-weighted ambiguities}

In this section we will analyse the variance matrix of the ambiguities for the case where the ionosphere is weighted. It will be clear, when the ionospheric delays are weighted, that then an ambiguity variance matrix $Q_{\hat{a}}(I)$ is obtained which 'interpolates' between $Q_{\hat{a} \mid I}$ and $Q_{\hat{a}}$. That is,

$Q_{\hat{a} \mid I} \leq Q_{\hat{a}}(I) \leq Q_{\hat{a}}$

with $Q_{\hat{a} \mid I}=Q_{\hat{a}}(I)$ when infinite weights are used and $Q_{\hat{a}}=Q_{\hat{a}}(I)$ when zero weights are used. It is not yet clear, however, what particular form this 'interpolation' takes. This will therefore be the topic of the present section. As a result we will also be able to show by how much the area of the ambiguity search space will change when ionospheric weights are used.

\subsection{Variance matrix as weighted mean}

Since the inverse of the ambiguity-ionosphere variance matrix

$$
\left[\begin{array}{cc}
Q_{\hat{a}}(I) & Q_{\hat{a} \hat{I}}(I) \\
Q_{\hat{I} \hat{a}}(I) & Q_{\hat{I}}(I)
\end{array}\right]
$$

is given as

$$
\left[\begin{array}{cc}
Q_{\hat{a} \mid I}^{-1} & -Q_{\hat{a}}^{-1} Q_{\hat{a} \hat{I}} Q_{\hat{I} \mid a}^{-1} \\
-Q_{\hat{I} \mid a}^{-1} Q_{\hat{I} \hat{a}} Q_{\hat{a}}^{-1} & {\left[Q_{\hat{I} \mid a}^{-1}+s_{I}^{-2} R_{I}^{-1}\right.}
\end{array}\right]
$$

it follows after reducing for the ionosphere and upon inversion that

$Q_{\hat{a}}(I)=\left[Q_{\hat{a} \mid I}^{-1}-Q_{\hat{a}}^{-1} Q_{\hat{a} \hat{I}} Q_{\hat{I} \mid a}^{-1}\left[Q_{\hat{I} \mid a}^{-1}+s_{I}^{-2} R_{I}^{-1}\right]^{-1} Q_{\hat{I} \mid a}^{-1} Q_{\hat{I} \hat{a}} Q_{\hat{a}}^{-1}\right]^{-1}$

We know that $Q_{\hat{I} \mid a}$ is a scaled unit matrix. We also know that in the ionosphere-float case, the covariances 
between the ambiguities and the ionosphere are constant in time. This implies that the matrix $Q_{\hat{a}}^{-1} Q_{\hat{a} \hat{l}} Q_{\hat{I} \mid a}^{-1}$ is a rank-1 matrix, which can be written as

$Q_{\hat{a}}^{-1} Q_{\hat{a} \hat{I}} Q_{\hat{I} \mid a}^{-1}=\frac{1}{k} c e_{k}^{T}$

This, combined with the previous equation, shows that

$$
\begin{aligned}
Q_{\hat{a}}(I) & =\left[Q_{\hat{a} \mid I}^{-1}-\sigma_{\bar{I} \mid a}^{2}(I) c c^{T}\right]^{-1} \\
& =Q_{\hat{a} \mid I}+Q_{\hat{a} \mid I} c\left[\sigma_{\bar{I} \mid a}^{-2}(I)-c^{T} Q_{\hat{a} \mid I} c\right]^{-1} c^{T} Q_{\hat{a} \mid I}
\end{aligned}
$$

In order to determine $c^{T} Q_{\hat{a} \mid I} c$, we first note that $Q_{\hat{a}}^{-1} Q_{\hat{a} \hat{I}} Q_{\hat{I} \mid a}^{-1}=Q_{\hat{a} \mid I}^{-1} Q_{\hat{a} \hat{I}} Q_{\hat{I}}^{-1}$. Hence

$$
\begin{aligned}
c^{T} Q_{\hat{a} \mid I} c & =e_{k}^{T} Q_{\hat{I}}^{-1} Q_{\hat{I} \hat{a}} Q_{\hat{a} \mid I}^{-1} \cdot Q_{\hat{a} \mid I} \cdot Q_{\hat{a}}^{-1} Q_{\hat{a} \hat{I}} Q_{\hat{I} \mid a}^{-1} e_{k} \\
& =e_{k}^{T} Q_{\hat{I}}^{-1}\left[Q_{\hat{I}}-Q_{\hat{I} \mid a}\right] Q_{\hat{I} \mid a}^{-1} e_{k}
\end{aligned}
$$

From substituting into this equation the ionospheric variance matrices Eqs. (23) and (28), we obtain

$c^{T} Q_{\hat{a} \mid I} c=\sigma_{\bar{I} \mid a}^{-2}-\sigma_{\bar{I}}^{-2}$

This result combined with Eq. (46) and using Eq. (39), gives

$Q_{\hat{a}}(I)=Q_{\hat{a} \mid I}+\sigma_{\bar{I}}^{2}(I) Q_{\hat{a} \mid I} c c^{T} Q_{\hat{a} \mid I}$

which shows that the ionosphere-weighted ambiguity variance matrix is a rank-1 update of the ionospherefixed ambiguity variance matrix. By taking infinite ionospheric weights in the preceding equation, we obtain

$Q_{\hat{a}}=Q_{\hat{a} \mid I}+\sigma_{\bar{I}}^{2} Q_{\hat{a} \mid I} c c^{T} Q_{\hat{a} \mid I}$

If we now combine this equation with the previous one, we finally are able to express the ionosphere-weighted ambiguity variance matrix in terms of the ionospherefixed and ionosphere-floated ambiguity variance matrices

$Q_{\hat{a}}(I)=\left(1-\frac{\sigma_{\bar{I}}^{2}(I)}{\sigma_{\bar{I}}^{2}}\right) Q_{\hat{a} \mid I}+\frac{\sigma_{\bar{I}}^{2}(I)}{\sigma_{\bar{I}}^{2}} Q_{\hat{a}}$

This is a remarkable result, which furthermore has been obtained without any assumptions on the structure of $s_{I}^{2} R_{I}$. It shows that $Q_{\hat{a}}(I)$ is simply a weighted mean of $Q_{\hat{a} \mid I}$ and $Q_{\hat{a}}$. Moreover, the weights are scalars, which implies that

$Z^{T} Q_{\hat{a}}(I) Z=\left(1-\frac{\sigma_{\bar{I}}^{2}(I)}{\sigma_{\bar{I}}^{2}}\right) Z^{T} Q_{\hat{a} \mid I} Z+\frac{\sigma_{\bar{I}}^{2}(I)}{\sigma_{\bar{I}}^{2}} Z^{T} Q_{\hat{a}} Z$

Hence the precision of every ambiguity function, such as for instance the widelane ambiguity or any one of the ambiguities which are obtained with the LAMBDA method, is weighted in an identical manner.

Equation 49 also shows that the weights are uniquely determined by the ionospheric variance ratio $\sigma_{\bar{I}}^{2}(I) / \sigma_{\bar{I}}^{2}$. This implies that the impact of the a priori ionospheric variance matrix $s_{I}^{2} R_{I}$, is only felt through the single scalar $\sigma_{\bar{I}}^{2}(I)$. This also implies that the equation can be used as a simple tool to infer a priori whether one is likely to have a successful ambiguity validation or not. Since $\sigma_{\bar{I}}^{2}$ and both $Q_{\hat{a} \mid I}$ and $Q_{\hat{a}}$ are known, the only free parameter left is $\sigma_{\bar{I}}^{2}(I)$.

\subsubsection{Impact of time correlation}

In order to study the impact of time correlation on the weights of Eq. (49), we can now make use of Eqs. 44 and 45. As a result we get for small time correlations

$$
\begin{aligned}
Q_{\hat{a}}(I) & =\frac{\sigma_{I}^{2}}{\sigma_{I}^{2}+s_{I}^{2}}\left[1-2 \rho_{t}\left(1-\frac{1}{k}\right) \frac{s_{I}^{2}}{\sigma_{I}^{2}+s_{I}^{2}}\right] Q_{\hat{a} \mid I} \\
& +\frac{s_{I}^{2}}{\sigma_{I}^{2}+s_{I}^{2}}\left[1+2 \rho_{t}\left(1-\frac{1}{k}\right) \frac{\sigma_{I}^{2}}{\sigma_{I}^{2}+s_{I}^{2}}\right] Q_{\hat{a}}
\end{aligned}
$$

This shows that in the presence of time correlation more weight is given to $Q_{\hat{a}}$ than to $Q_{\hat{a} \mid I}$. Note that the weights reach a steady state when $k$ is large enough. In case time correlation is absent though, the weights become completely independent of the number of samples used. In that case we have

$Q_{\hat{a}}(I)=\frac{\sigma_{I}^{2} Q_{\hat{a} \mid I}+s_{I}^{2} Q_{\hat{a}}}{\sigma_{I}^{2}+s_{I}^{2}}$

Hence, like $Q_{\hat{a} \mid I}$ and $Q_{\hat{a}}$, also $Q_{\hat{a}}(I)$ is then inversely proportional to $k$. The equation also shows that it is the code variance which competes with the spatial correlation in determining the contribution of the two variance matrices to the final result. The longer the baseline, the more the weight given to $Q_{\hat{a}}$.

Analogous to Eq. (50), we obtain for large time correlations

$$
\begin{aligned}
Q_{\hat{a}}(I) & =\frac{\sigma_{I}^{2}}{\frac{1}{k} \sigma_{I}^{2}+s_{I}^{2}}\left[\frac{1}{k}+\frac{1}{3}\left(1-\rho_{t}\right)\left(1-\frac{1}{k^{2}}\right) \frac{s_{I}^{2}}{\frac{1}{k} \sigma_{I}^{2}+s_{I}^{2}}\right] Q_{\hat{a} \mid I} \\
& +\frac{s_{I}^{2}}{\frac{1}{k} \sigma_{I}^{2}+s_{I}^{2}}\left[1-\frac{1}{3}\left(1-\rho_{t}\right)\left(1-\frac{1}{k^{2}}\right) \frac{\sigma_{I}^{2}}{\frac{1}{k} \sigma_{I}^{2}+s_{I}^{2}}\right] Q_{\hat{a}}
\end{aligned}
$$

This simplifies to

$Q_{\hat{a}}(I)=\frac{\sigma_{I}^{2} Q_{\hat{a} \mid I}+k s_{I}^{2} Q_{\hat{a}}}{\sigma_{I}^{2}+k s_{I}^{2}}$

in the case where time correlation is at its maximum. This shows that as the number of samples increases, more weight is given to the ionosphere-float solution. In fact for $k$ large enough, we have the approximation $Q_{\hat{a}}(I) \simeq Q_{\hat{a}}$.

\subsection{The area of the search space}

In Sect. 4, the determinant of the ionosphere-fixed ambiguity variance matrix was shown to be given as

$\left|Q_{\hat{a} \mid I}\right|=\frac{\sigma_{\phi_{1}}^{2} \sigma_{\phi_{2}}^{2}}{\lambda_{1}^{2} \lambda_{2}^{2} k^{2}}\left(1+\sigma_{p_{w}}^{2} / \sigma_{\phi_{w}}^{2}\right)$ 
and in Sect. 5.2, its ionosphere-floated counterpart was given as

$$
\left|Q_{\hat{a}}\right|=\left|Q_{\hat{a} \mid I}\right| \frac{\sigma_{I}^{2}}{\sigma_{I \mid a}^{2}}
$$

We can now use Eq. (48) to generalize this result to the ionosphere-weighted case. Upon taking the determinant of Eq. (48) we get

$$
\begin{aligned}
\left|Q_{\hat{a}}(I)\right| & =\left|Q_{\hat{a} \mid I}\right|\left|I_{2}+\sigma_{\bar{I}}^{2}(I) c c^{T} Q_{\hat{a} \mid I}\right| \\
& =\left|Q_{\hat{a} \mid I}\right|\left(1+\sigma_{\bar{I}}^{2}(I) c^{T} Q_{\hat{a} \mid I} c\right)
\end{aligned}
$$

which reduces with Eq. (47) to

$$
\left|Q_{\hat{a}}(I)\right|=\left|Q_{\hat{a} \mid I}\right| \frac{\sigma_{\bar{I}}^{2}(I)}{\sigma_{\bar{I} \mid a}^{2}(I)}
$$

This shows that when the ionosphere is weighted instead of fixed, the area of the search space is enlarged by a factor equal to the square root of the ratio of the variances of the time-averaged ionosphere before and after ambiguity fixing. It can be shown that this ratio is also the largest generalized eigenvalue of $\mid Q_{\hat{a}}(I)-$ $\lambda Q_{\hat{a} \mid I} \mid=0$. Hence, it equals the largest factor by which the precision of the ambiguities degrades due to a weighting instead of a fixing of the ionosphere.

In order to express this result in terms of $s_{I}^{2}$ (spatial decorrelation) and $\rho_{t}$ (time correlation), we can make use of Eqs. 44 and 45. As a result we obtain for the case where the time correlation is small

$$
\frac{\left|Q_{\hat{a}}(I)\right|}{\left|Q_{\hat{a} \mid I}\right|}=\frac{1+\frac{s_{I}^{2}}{\sigma_{I \mid a}^{2}}}{1+\frac{s_{I}^{2}}{\sigma_{I}^{2}}}\left[1+2 \rho_{t} \frac{\left(1-\frac{1}{k}\right)\left(\frac{s_{I}^{2}}{\sigma_{I \mid a}^{2}}-\frac{s_{I}^{2}}{\sigma_{I}^{I}}\right)}{\left(1+\frac{s_{I}^{2}}{\sigma_{I \mid a}}\right)\left(1+\frac{s_{I}^{2}}{\sigma_{I}^{2}}\right)}\right]
$$

and for the case where the time correlation is large, we get

$$
\frac{\left|Q_{\hat{a}}(I)\right|}{\left|Q_{\hat{a} \mid I}\right|}=\frac{\frac{1}{k}+\frac{s_{I}^{2}}{\sigma_{I \mid a}^{2}}}{\frac{1}{k}+\frac{s_{I}^{2}}{\sigma_{I}^{2}}}\left[1-\frac{1}{3}\left(1-\rho_{t}\right) \frac{\left(1-\frac{1}{k^{2}}\right)\left(\frac{s_{I}^{2}}{\sigma_{I \mid a}^{2}}-\frac{s_{I}^{2}}{\sigma_{I}^{2}}\right)}{\left(\frac{1}{k}+\frac{s_{I}^{2}}{\sigma_{I \mid a}^{2}}\right)\left(\frac{1}{k}+\frac{s_{I}^{2}}{\sigma_{I}^{2}}\right)}\right]
$$

Note that in both cases, the ratio of the two areas reaches a steady state when $k$ is sufficiently large. Also note that it is not so much the absolute value of $s_{I}^{2}$ that determines by how much the area gets blown up, as its value relative to the precision with which the ionosphere can be estimated, before and after ambiguity fixing when the ionosphere is floated. Thus it is the two variance ratios

$\frac{s_{I}^{2}}{\sigma_{I \mid a}^{2}}$ and $\frac{s_{I}^{2}}{\sigma_{I}^{2}}$

that count. It is also through these two ratios that the impact of the precision of the GPS observables is felt. The variance $\sigma_{I \mid a}^{2}$ is namely predominantly determined by the variances of the phase data, while $\sigma_{I}^{2}$ is only determined by the variances of the code data.
The preceding results reduce to Eq. (54), when the spatial decorrelation is at its minimum, $s_{I}^{2}=0$, and to Eq. (55), when $s_{I}^{2}=\infty$. For the case where time correlation is at its minimum $\left(\rho_{t}=0\right)$ or at its maximum $\left(\rho_{t}=1\right)$, we get

$$
\begin{aligned}
& \left|Q_{\hat{a}}\left(I, \rho_{t}=0\right)\right|=\left|Q_{\hat{a} \mid I}\right|\left(1+\frac{s_{I}^{2}}{\sigma_{I \mid a}^{2}}\right) /\left(1+\frac{s_{I}^{2}}{\sigma_{I}^{2}}\right) \\
& \left|Q_{\hat{a}}\left(I, \rho_{t}=1\right)\right|=\left|Q_{\hat{a} \mid I}\right|\left(\frac{1}{k}+\frac{s_{I}^{2}}{\sigma_{I \mid a}^{2}}\right) /\left(\frac{1}{k}+\frac{s_{I}^{2}}{\sigma_{I}^{2}}\right)
\end{aligned}
$$

\section{Summary}

In this contribution we analysed the ambiguity search space of the geometry-free model for the general case that the ionospheric delays are a priori weighted. It was shown how the ambiguity search space was affected by the use of these a priori weights. In order to obtain a qualitative description, analytical rather than numerical results were emphasized. Due to the general form of the chosen a priori weights, our results are applicable to the whole range of baseline lengths that one may encounter in practice. For short baselines for instance, one will generally choose infinite weights. This implies that one assumes the ionospheric delays to be either absent or known. For very long baselines however, the weights are put to zero. This implies that the ionospheric delays are assumed to be completely unknown. And for the intermediate cases, non-zero but finite weights can be used.

Since we have the well-known inequality relation

$Q_{\hat{a} \mid I} \leq Q_{\hat{a}}(I) \leq Q_{\hat{a}}$

we started off studying the two extreme cases, namely the ionosphere-fixed case $Q_{\hat{a} \mid I}$, and the ionospherefloated case, $Q_{\hat{a}}$. The dependency of the ambiguities and the shape of the ambiguity search space was described by, respectively, the correlation coefficient, the orientation and the elongation. Expressions for these dimensionless diagnostics were derived and it was shown that they all are governed by the very small phase-code variance ratio $\sigma_{\phi}^{2} / \sigma_{p}^{2}$. As a result we have, for both the ionosphere-fixed and for the ionosphere-floated case, that the ambiguities are highly correlated and that their search space is very elongated. The two cases differ however with respect to their sensitivity to changes in the phase-code variance ratio. In particular the correlation and orientation was shown to be rather insensitive to changes in the phase-code variance ratio in case the ionosphere was floated.

To what extent the ionosphere-weighted ambiguity variance matrix interpolates between its fixed and floated counterparts was made precise by showing that

$$
Q_{\hat{a}}(I)=\left(1-\frac{\sigma_{\bar{I}}^{2}(I)}{\sigma_{\bar{I}}^{2}}\right) Q_{\hat{a} \mid I}+\frac{\sigma_{\bar{I}}^{2}(I)}{\sigma_{\bar{I}}^{2}} Q_{\hat{a}}
$$


This is a weighted mean, with the scalar weights determined by the variance ratio $\sigma_{\bar{I}}^{2}(I) / \sigma_{\bar{I}}^{2}$. Its numerator is the variance of the time-averaged ionosphere when the ionosphere is a priori weighted, and its denominator is the variance of the time-averaged ionosphere when the ionosphere is kept floated. This variance ratio could be expressed in two other variance ratios as

$\frac{\sigma_{\bar{I}}^{2}(I)}{\sigma_{\bar{I}}^{2}}=\left(1+\frac{\sigma_{\bar{I}}^{2}}{\sigma_{\bar{I} \mid a}^{2}}\left[\frac{\sigma_{\bar{I} \mid a}^{2}}{\sigma_{\bar{I} \mid a}^{2}(I)}-1\right]\right)^{-1}$

The denominator and numerator of the variance ratio $\sigma_{\bar{I}}^{2} / \sigma_{\bar{I} \mid a}^{2}$ were given as

$\sigma_{\bar{I} \mid a}^{2}=\frac{1}{k} \sigma_{I \mid a}^{2} \simeq \frac{1}{k} \frac{2 \sigma_{\phi}^{2}}{\left(\mu_{1}-\mu_{2}\right)^{2}}$

$\sigma_{\bar{I}}^{2}=\frac{1}{k} \sigma_{I}^{2}=\frac{1}{k} \frac{2 \sigma_{p}^{2}}{\left(\mu_{1}-\mu_{2}\right)^{2}}$

These are the variances of the a priori unweighted timeaveraged ionosphere for the case where the ambiguities are fixed and floated. It is through these two variances that the impact of the precision of the GPS observables is felt. The first variance is predominantly governed by the precision of the phase observables and therefore very small, whereas the second variance only depends on the variance of the code observables.

Besides the impact of the precision of the GPS observables, we also have the impact of the a priori chosen weights of the ionospheric delays. It is felt through the second variance ratio

$$
\frac{\sigma_{\bar{I} \mid a}^{2}}{\sigma_{\bar{I} \mid a}^{2}(I)}=\left(1-\frac{1}{k} e_{k}^{T}\left[I_{k}+\frac{s_{I}^{2}}{\sigma_{I \mid a}^{2}} R_{I}\right]^{-1} e_{k}\right)^{-1}
$$

where $s_{I}^{2} R_{I}$ is the a priori ionospheric variance matrix, factored in a spatial decorrelation dependent variance factor $s_{I}^{2}$ and a time correlation dependent correlation matrix $R_{I}$.

In order to obtain the impact of the a priori chosen weights, we considered different correlation lengths for the time-dependent behaviour of the ionosphere. In order to have an easy reference, they are summarized in Table 1 for the time-averaged ionosphere. It was also shown how these results propagate into, for instance, the weighted mean already seen. And one of the conclusions was, since the precision of the estimators de- grades when the time correlation increases, that for a fixed number of samples one is forced to use a lower sampling rate in order to handle longer baselines and still obtain the same level of precision.

Apart from the shape of the ambiguity search space, we also considered its size in terms of the search-space area. The relevance of the area is that it gives an indication of the number of grid points that are located inside the search space. The determinant of the ambiguity variance matrix and thus also the search-space area combine the ambiguity precision together with the ambiguity correlation, and it is invariant for any one of the admissible ambiguity transformations. As with the inequality relation already given, we have

$$
\left|Q_{\hat{a} \mid I}\right| \leq\left|Q_{\hat{a}}(I)\right| \leq\left|Q_{\hat{a}}\right|
$$

The determinant of the ambiguity variance matrix in case the ionosphere is fixed was shown to be given as

$\left|Q_{\hat{a} \mid I}\right|=\frac{\sigma_{\phi_{1}}^{2} \sigma_{\phi_{2}}^{2}}{\lambda_{1}^{2} \lambda_{2}^{2} k^{2}}\left(1+\sigma_{p_{w}}^{2} / \sigma_{\phi_{w}}^{2}\right)$

The determinant and thus the area of the search space blow up however, when instead of infinite weights, finite weights are used. The relation between the two determinants was shown to be given as

$$
\left|Q_{\hat{a}}(I)\right|=\left|Q_{\hat{a} \mid I}\right| \frac{\sigma_{\bar{I}}^{2}(I)}{\sigma_{\bar{I} \mid a}^{2}(I)}
$$

The dependency on spatial correlation and on time correlation of the factor by which the area blows up, follows then directly from the results of Table 1 . This multiplication factor reaches its maximum when zero weights are used. In that case, it equals $\sigma_{I}^{2} / \sigma_{I \mid a}^{2}$, which is approximately equal to the reciprocal of the phase-code variance ratio. The conclusion was therefore reached that the smallest determinant in the above inequality relation, is governed by the product of the code variance with the phase variance, while the largest determinant is governed by the product of the code variance with itself.

Acknowledgements. This work was supported by the Alexander von Humboldt Stiftung and finalized during the author's sabattical stay at the University of Stuttgart (Germany), with Professor E.W. Grafarend as his host.

Table 1. The precision of the time-averaged ionosphere, using $s_{I}^{2} R_{I}$ as a priori variance matrix, with $R_{I}=\left[\rho_{t}^{|i-j|}\right]$

\begin{tabular}{lll}
\hline & $\sigma_{\bar{I} \mid a}^{2}(I)$ & $\sigma_{\bar{I}}^{2}(I)$ \\
\hline$\rho_{t}=0$ & $\frac{1}{k} \frac{\sigma_{I \mid a}^{2} s_{I}^{2}}{\sigma_{I \mid a}^{2}+s_{I}^{2}}$ & $\frac{1}{k} \frac{\sigma_{I}^{2} s_{I}^{2}}{\sigma_{I}^{2}+s_{I}^{2}}$ \\
$\rho_{t}=$ small & $\sigma_{\bar{I} \mid a}^{2}\left(I, \rho_{t}=0\right)\left[1+2 \rho_{t}\left(1-\frac{1}{k}\right) \frac{\sigma_{I \mid a}^{2}}{\sigma_{I \mid a}^{2}+s_{I}^{2}}\right]$ & $\sigma_{\bar{I}}^{2}\left(I, \rho_{t}=0\right)\left[1+2 \rho_{t}\left(1-\frac{1}{k}\right) \frac{\sigma_{I}^{2}}{\sigma_{I}^{2}+s_{I}^{2}}\right]$ \\
$\rho_{t}=$ large & $\sigma_{\bar{I} \mid a}^{2}\left(I, \rho_{t}=1\right)\left[1-\frac{1}{3}\left(1-\rho_{t}\right)\left(k-\frac{1}{k}\right) \frac{\sigma_{I \mid a}^{2}}{\sigma_{I \mid a}^{2}+k s_{I}^{2}}\right]$ & $\sigma_{\bar{I}}^{2}\left(I, \rho_{t}=1\right)\left[1-\frac{1}{3}\left(1-\rho_{t}\right)\left(k-\frac{1}{k}\right) \frac{\sigma_{I}^{2}}{\sigma_{I}^{2}+k s_{I}^{2}}\right]$ \\
$\rho_{t}=1$ & $\frac{1}{k} \frac{\sigma_{I \mid a}^{2} k s_{I}^{2}}{\sigma_{I \mid a}^{2}+k s_{I}^{2}}$ & $\frac{1}{k} \frac{\sigma_{I}^{2} k s_{I}^{2}}{\sigma_{I}^{2}+k s_{I}^{2}}$ \\
\hline
\end{tabular}




\section{References}

Bock Y (1996) Medium-distance GPS measurements. In: Kleusberg A, Teunissen PJG (eds) GPS for Geodesy, Lecture Notes in Earth Sciences, vol 60. Springer, Berlin Heidelberg New York, Chap 9

Dedes G, Goad C (1994) Real-time cm-level GPS positioning of cutting blade and earth moving equipment. In: Proc 1994 Nat Tech Meeting ION, San Diego, California, pp 587-594

De Jonge PJ, Tiberius CCJM (1996) The LAMBDA method for integer ambiguity estimation: implementation aspects. $L G R$ Ser 12, Delft University of Technology, Delft

Delikaraoglou D (1989) On the stochastic modelling of GPS ionospheric delays. Manuscr Geod 14:100-109

Euler H-J, Goad C (1990) On optimal filtering of GPS dual-frequency observations without using orbit information. Bull Geod 65: 130-143

Georgiadou Y (1994) Modelling the ionosphere for an active control network of GPS stations. LGR-Ser 7, Delft University of Technology, Delft

Goad C (1990) Optimal filtering of pseudo-ranges and phases from single-frequency GPS receivers. Navigation 37: 365-369

Hatch R (1982) The synergism of GPS code and carrier phase measurements. In: Proc 3rd Int Geod Symp Satellite Positioning, Las Cruces, New Mexico, 8-12 February, 1982, vol 2. pp 12131231
Schaer S (1994) Stochastische Ionospharenmodellierung beim "Rapid Static Positioning" mit GPS. Astronomisches Institut, Universität Bern

Teunissen PJG (1993) Least-squares estimation of the integer GPS ambiguities. LGR Ser 6, Delft University of Technology, Delft

Teunissen PJG (1995) The least-squares ambiguity decorrelation adjustment: a method for fast GPS integer ambiguity estimation. J Geod 70: 65-82

Teunissen PJG (1996) An analytical study of ambiguity decorrelation using dual-frequency code and carrier phase. J Geod 70: 515-528

Teunissen PJG, de Jonge PJ, Tiberius CCJM (1996) The volume of the GPS ambiguity search space and its relevance for integer ambiguity resolution. In: Proc ION-96. pp: 889-898

Wanninger L (1995) Improved ambiguity resolution by regional differential modelling of the ionosphere. In: Proc ION GPS-95 12-15 September 1995, Palm Springs. pp 889-898

Wild U (1994) Ionosphere and geodetic satellite systems: permanent GPS tracking data for modelling and monitoring. GeodGeophys Arb Schweiz, Band 48

Wild U, Beutler G (1991) Deterministische und stochastische Ionospharenmodelle. Z Vermess Photogramm Kulturtech 6: 298-302

Wübbena G (1988) GPS carrier phases and clock modeling. In: Groten E, Strauss R (eds) GPS-Techniques Applied to Geodesy and Surveying. Lecture Notes in Earth Sciences vol 19. Springer, Berlin Heidelberg New York, pp 381-392 